\title{
Hernia of Morgagni
}

\author{
F. PAR ÍS, V. TARAZONA, M. CASILLAS, E. B LASCO, \\ A. CANTÓ, J. PASTOR, and A. ACOSTA \\ Thoracic Surgery Service, Department of Surgery, Centro Hospitalario 'La Fé', Valencia 9, Spain
}

\begin{abstract}
París, F., Tarazona, V., Casillas, M., Blasco, E., Cantó, A., Pastor, J., and Acosta, A. (1973). Thorax, 28, 631-636. Hernia of Morgagni. Nine patients with Margagni's hernia of the diaphragm are reported, seven of whom have been operated on. Four patients had gastrointestinal symptoms, two respiratory symptoms, and one retrosternal pain; the other two were asymptomatic. In the first case the diagnosis was made at the time of thoracotomy, in the other eight it was suggested by radiological studies, The standard midline epigastric incision was used for repair in four cases and thoracotomy in two; the last patient was operated on through a preperitoneal subxiphoid route. This route has the advantage of not requiring a wide incision of peritoneum.
\end{abstract}

The passage of abdominal contents into the thoracic cavity through an anterior retrosternal diaphragmatic defect has been known as a hernia of Morgagni, according to Morgagni's (1761) first description.

There is, however, certain confusion on the name. Some authors apply Larrey's name to this type of hernia; others use Morgagni for rightsided hernia and Larrey's for left, while others use the names less discriminatingly. In order to clarify this apparent confusion it must be recalled that Larrey (1829) never described a hernia. He only developed a surgical approach to the pericardial cavity.

Therefore, the anterior diaphragmatic hernia, whether right or left, should be referred to as the hernia of Morgagni, or hernia through Larrey's space. Other anatomical terms are used, such as subcostosternal hernia (Harrington, 1951), retrocostoxiphoid hernia (Denisart, 1951), retrosternal hernia (Warwick-Brown, 1953), parasternal hernia (Chin and Duchesne, 1955) or simply anterior diaphragmatic hernia (Dor and Eymery, 1956).

\section{ANATOMY AND PATHOLOGY}

The development of the pars muscularis of the diaphragm occurs after the fusion of the septum transversum with the pleuroperitoneal folds and the dorsal mesentery. The muscle fibres proceeding from the third, fourth, and fifth cervical myotomes develop between the folds of the primitive pleura and the peritoneum and complete the diaphragm at the ninth week of fetal life.

Three elements are differentiated in this muscularization-the pars lumbaris, costalis, and sternalis. The costalis is formed by the fibres spreading out from the tendinous centre to the last six costal arches. The pars sternalis is formed from the fibres going to the inner surface of the xiphoid process, a few of them spreading to the posterior aspect of the rectus abdominis sheath.

The failure of fusion between the fibrotendinous elements of the pars sternalis and those arising from the seven costochondral arches leaves a muscle-free area, the costosternale trigone, known as Larrey's space or Morgagni's foramen. Normally this space is filled with fat and covered with a layer of pleura above and peritoneum below. In the Morgagni hernia the peritoneum and the abdominal contents pass upwards through this space into the thoracic cavity.

Diaphragmatic hernia can be either congenital or acquired. Most of them are mixed. There is an embryonal failure of muscularization which will produce a weak area. Acquired factors, such as severe effort and obesity, will produce an increased intra-abdominal pressure and promote the development of a hernial sac.

The muscular defect may appear in different sites. The defect is usually lateral to the xiphoid process, especially on the right side. Sometimes it is bilateral. The absence of the pars sternalis produces a median defect posterior to the xiphoid pro- 
cess. The bilateral and median types combined give a larger defect resembling an aplasia of the anterior part of the diaphragm. A rare type is the combination of the diaphragmatic hernia with a pericardial defect.

Almost all Morgagni herniae have a sac, but occasionally this may be absent. Kelly and Bassett (1964) found a hernia of preperitoneal fat without
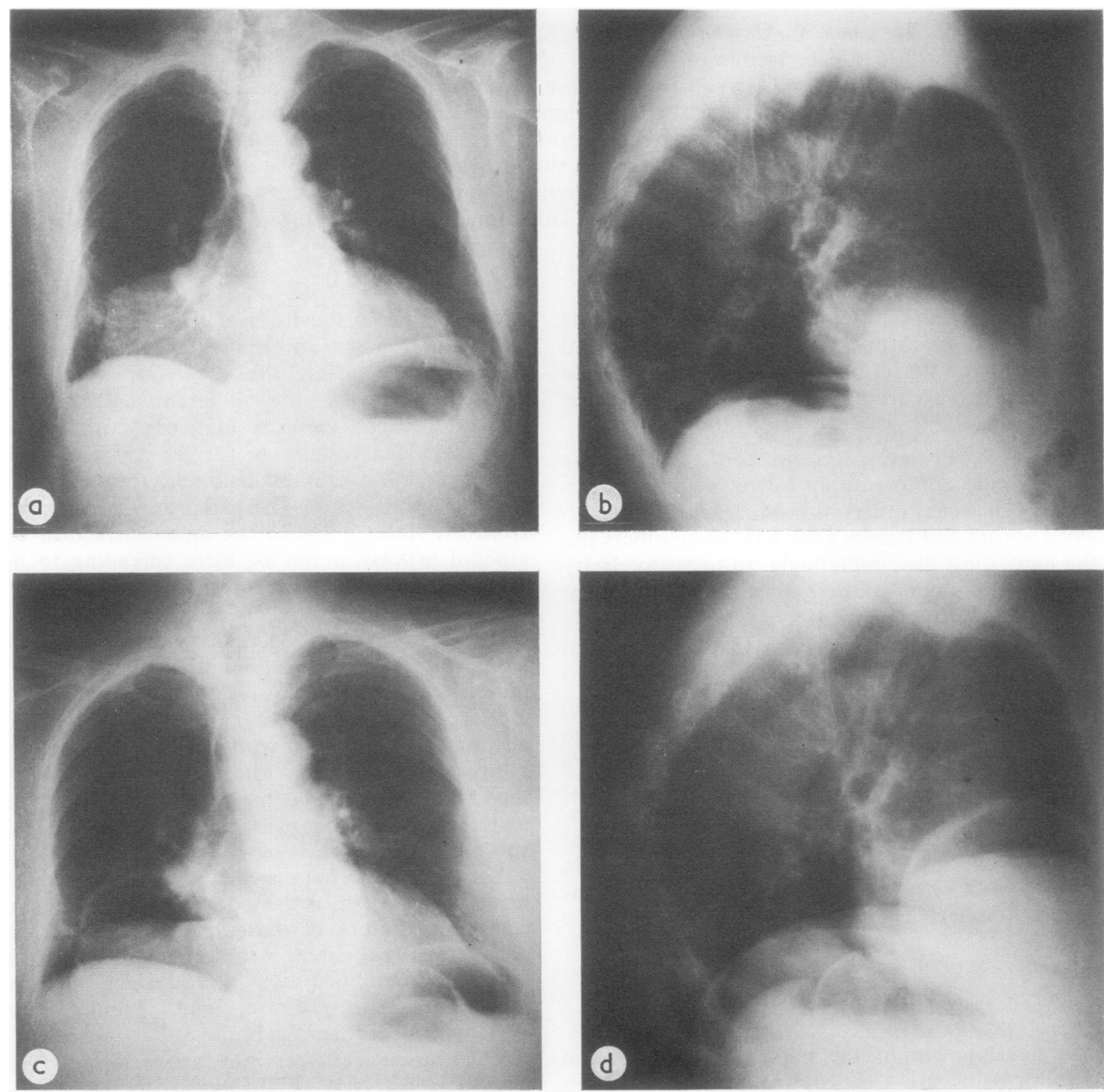

a sac, as did Comer and Clagett (1966) in four patients. The defect may rarely be more complex $\frac{\bar{\sigma}}{T}$ with a patent sternocostal foramen and an absence $\stackrel{\varnothing}{\Omega}$ of diaphragmatic tissue. Often the hernial sac contains only the colon, or the colon with either $\vec{P}$ omentum, stomach or small intestine. Sometimes it is filled only with omentum. The liver occasionally protrudes into the sac.

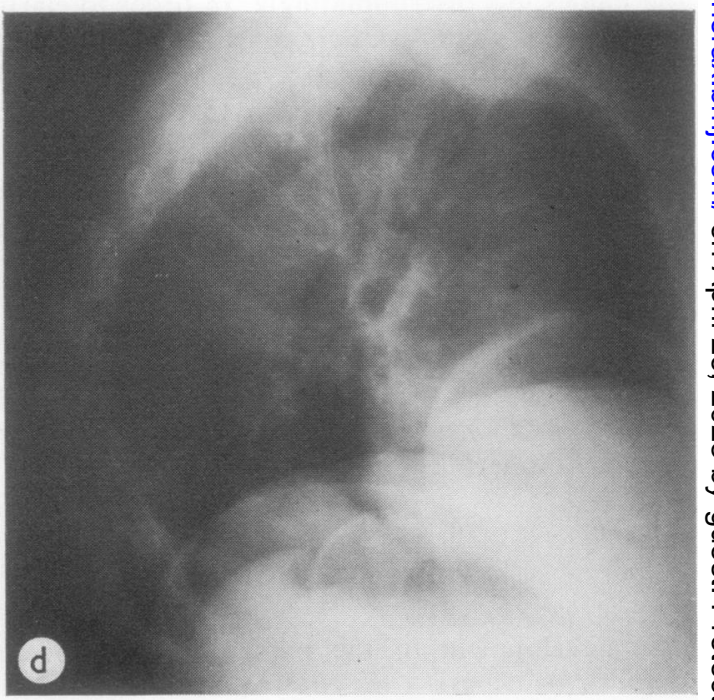

FIG. 1. Anteroposterior (a) and lateral (b) chest films in a 65-year-old man with a history of chronic bronchitis. There is a retrosternal opacity in the right cardiophrenic angle. The pneumoperitoneum $(c, d)$ fills the sac and confirms the diagnosis of Morgagni hernia. 

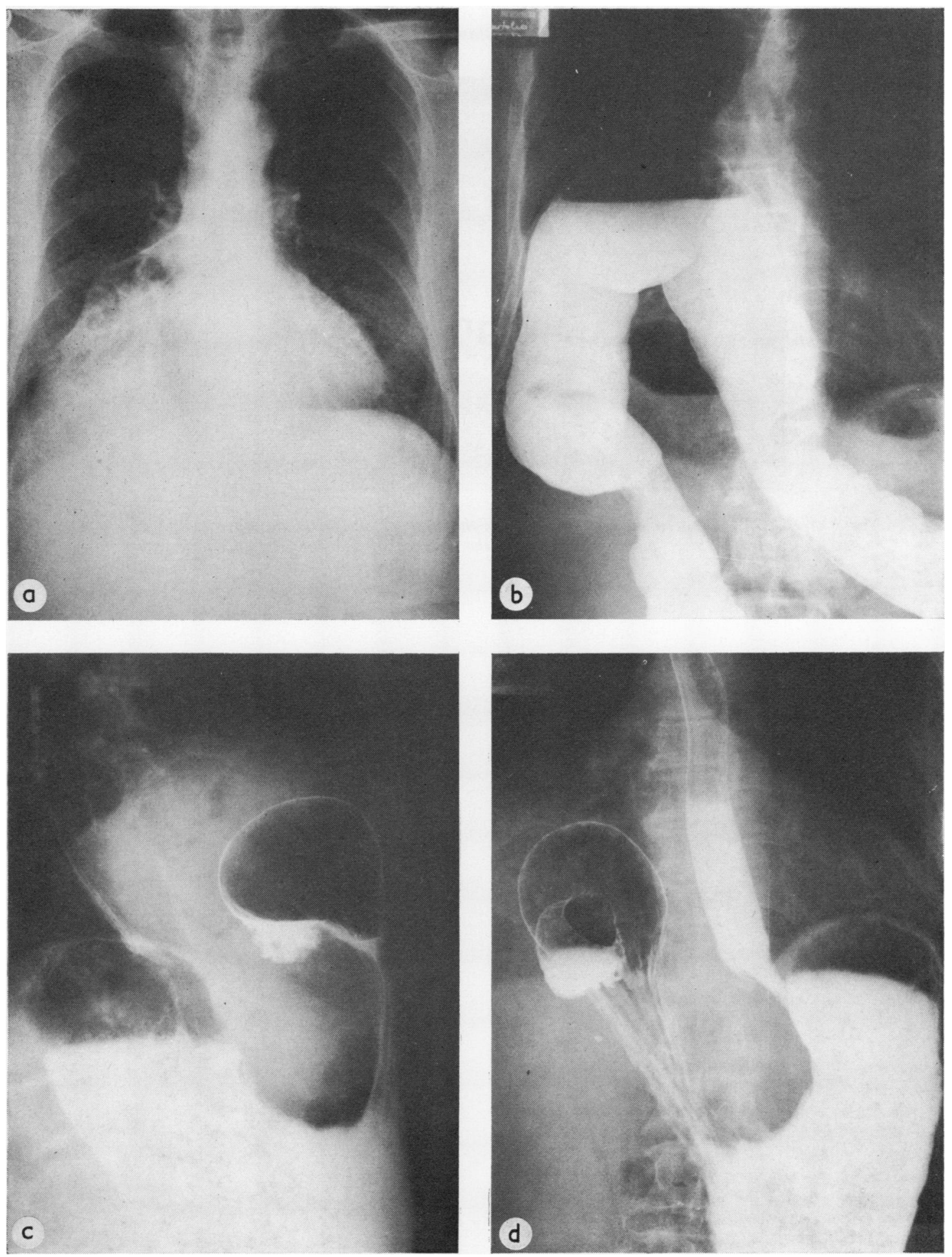

FIG. 2. Chest films in a 66-year-old patient with symptoms of severe nausea and vomiting. The anteroposterior film (a) shows the radiolucent area. The barium studies $(b, c, d)$ demonstrate the herniation of the stomach and colon into the peritoneal sac. 


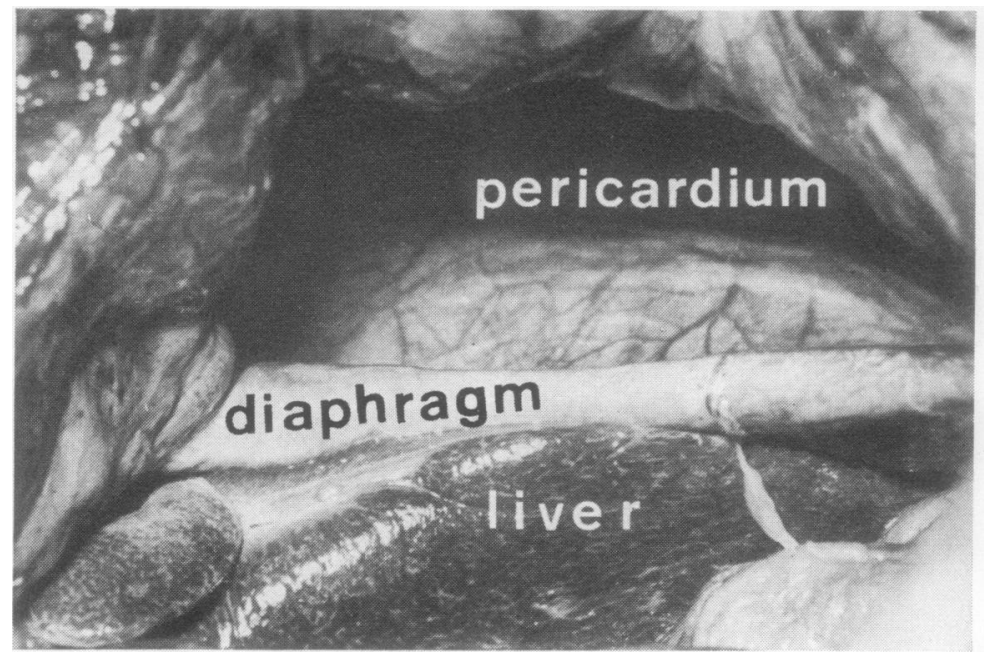

FIG. 3. Bilateral hernia operated on through an upper midline laparotomy. Note the large anterior defect and the absence of the pars sternalis fibres.
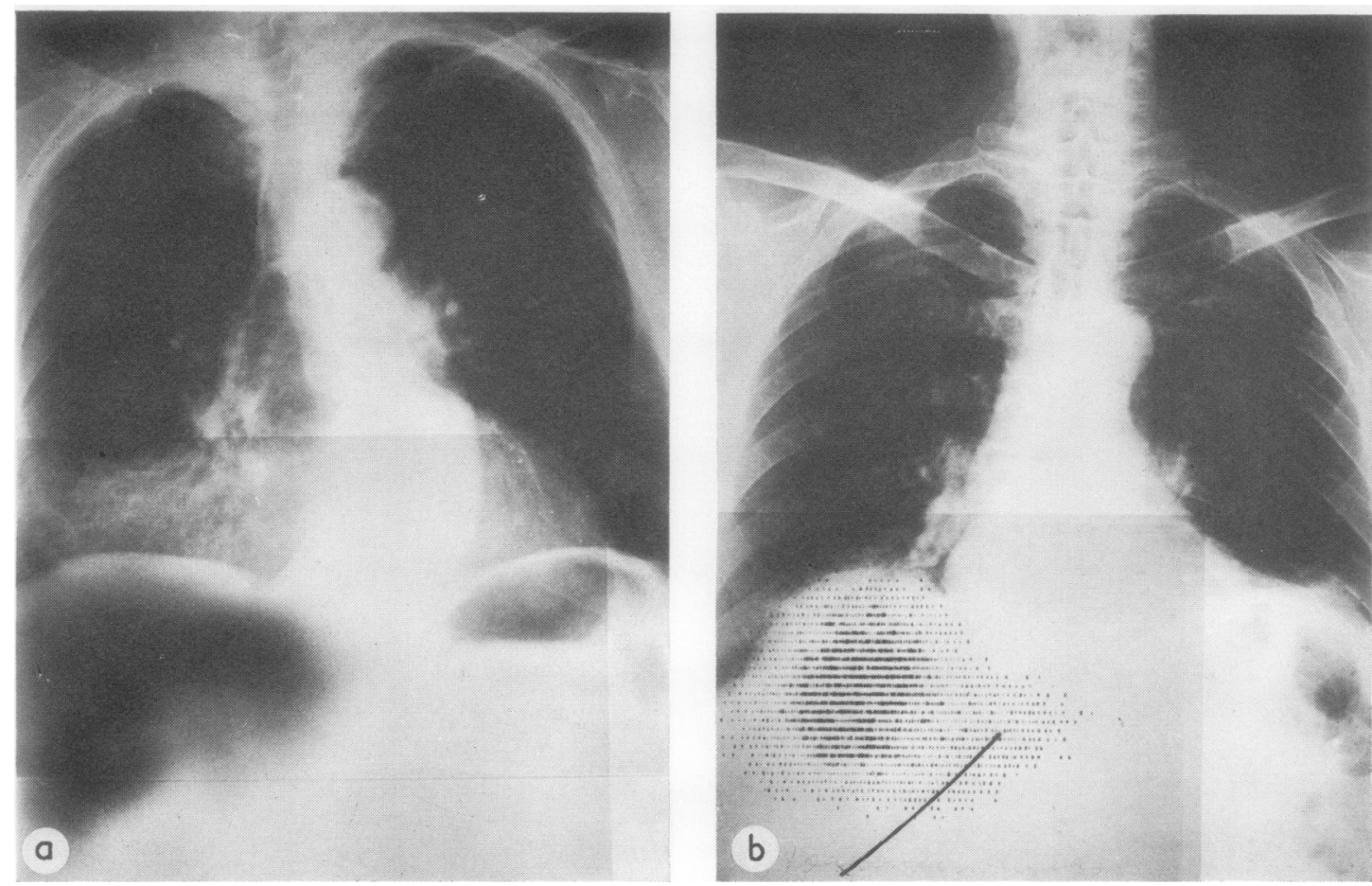

FIG. 4. Liver scan of a patient (a) with a Morgagni hernia containing only omentum. (b) Shows a partial eventration of the diaphragm (superimposition of the liver scan on to the anteroposterior chest film). 

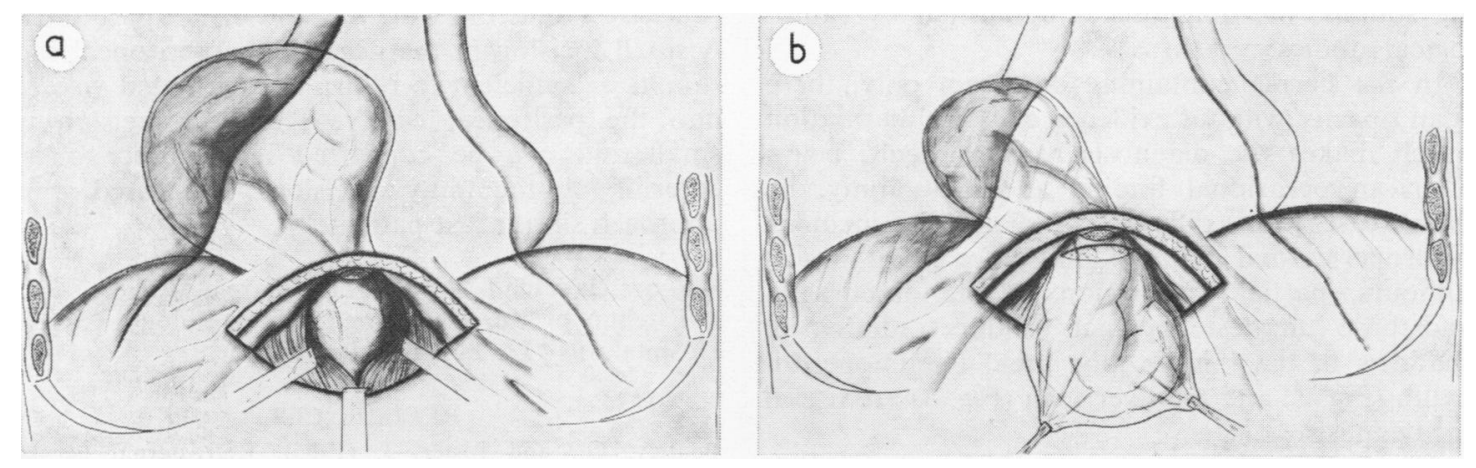

FIG. 5. Two views of proposed diaphragmatic exposure through a subxiphoid approach.

\section{CLINICAL MATERIAL}

During the last 11 years (1962-73) the authors have seen nine patients with a Morgagni hernia, six in adults and three in children. Two were infants of 12 and 18 months, and the third child was 8 years old. The adults were aged between 45 and 66 years. Seven were men.

When they were referred to us the diagnosis was correct in only two patients. Five patients were thought to have an intrathoracic tumour, one a pulmonary cyst, and the other a peptic stenosis of the duodenum. Four patients presented with gastrointestinal symptoms, such as nausea, vomiting, dull pain in the right subcostal area, and abdominal distension after taking food. Two had respiratory symptoms such as cough, expectoration and dyspnoea secondary to chronic bronchitis rather than to the hernia ; another gave a history of retrosternal pain. Two patients were asymptomatic and the diagnosis was disclosed by radiological study. One adult had a marked kyphoscoliosis, and another had umbilical and femoral herniae.

In the first patient the diagnosis was made only at the time of thoracotomy ; in the other eight it was suggested by the chest films. In three there was an opacity in the right cardiophrenic angle which was retrosternal in the lateral view (Fig. 1). In six cases the herniation of a hollow viscus gave a radiolucent shadow. Our working diagnosis was confirmed by barium studies (Fig. 2). Pneumoperitoneum was employed on three occasions (Fig. 1).

Seven patients (five adults and two infants) were operated on. The parents refused operation on one child. Surgical treatment was not considered in another high-risk case because of previous myocardial infarction and chronic bronchitis with cyanosis at rest.

The standard midline epigastric incision was used in four patients. A right thoracotomy was performed in two cases. The last patient was operated on through a preperitoneal subxiphoid route.

In six patients the hernia was right-sided, bilateral in three (Fig. 3). The colon was the main herniated viscus in six patients, although accompanied by small intestine in three and by a portion of the stomach in two; in another two patients the hernia contained omentum only. In one patient the preperitoneal fat was the only material herniated. The defect was closed by bringing up the anterior rim of the diaphragm to the retrosternal fascia, the costal margins, and the rectus sheath. Non-absorbable suture material was used.

\section{DISCUSSION}

Morgagni's herniae are uncommon. Harrington (1951) in 534 cases of diaphragmatic herniae found only 14 Morgagni's herniae. Chin and Duchesne (1955) found 30 cases in a mass radiography survey. Comer and Clagett (1966) described 50 cases operated on from 1,750 diaphragmatic herniae at the Mayo Clinic in 32 years, an incidence of $3 \%$. Recently, Thomas (1972) presented six cases in an eight-year period. In a review of the Spanish literature Martinez (1973) found 46 cases.

This condition affects the right side more frequently than the left. Comer and Clagett (1966) found only one left-sided and four bilateral herniae among their 50 patients. In one of our patients the diaphragmatic defect was large, extending bilaterally without intervening muscle fibres (Fig. 3), the type II described by Chin and Duchesne (1955). This type of hernia can be asymptomatic or cause symptoms of partial intestinal obstruction. The sudden entry of the stomach and duodenum into the sac may manifest itself dramatically. Respiratory symptoms may also be present but sometimes are not related to the hernia.

Although percussion and auscultation of the thorax may reveal areas of tympanism and splash- 
ing sounds, the diagnosis is confirmed by radiological studies.

In the hernia containing omentum only, there is an opacity without evidence of bowel herniation which makes the diagnosis more difficult, being often an occasional finding at thoracotomy. In these cases the differential diagnosis includes pleuropericardial cyst, lipoma, other intrathoracic tumours, partial eventration of the diaphragm, and those lung lesions which produce radiological shadows in the middle lobe area. Pneumoperitoneum (Fig. 1) and the liver scan (Fig. 4) are useful in diagnosis.

If a hollow viscus is herniated the diagnosis is easier. Chest films may show the haustrations of the colon or other gas pattern in the right cardiophrenic angle behind the sternum. A barium enema will usually confirm the diagnosis when the colon is herniated. A barium swallow and follow through may complete the study.

Generally treatment is surgical, to correct symptoms due to the hernia and prevent possible strangulation.

Most authors favour the abdominal route for closure as the defect is easily repaired. The withdrawal and resection of the sac presents no difficulties. If there are adhesions of the sac to the pericardium or pleura the neck may be sectioned and left in the mediastinum.

Chin and Duchesne (1955) advise the thoracic approach as it gives better access to the sac; the pericardial adhesions can be easily released, the diaphragmatic defect is easy to repair, and both the subcostal margin and the edge of the diaphragmatic foramen are accessible. They feel that the intra-abdominal approach may be difficult in obese patients and an intrathoracic sac may remain where a cyst could later develop.

Boyd (1961) operated on nine of his 10 cases by the thoracic approach but stated that if the diagnosis is correct the abdominal route must be preferred. Comer and Clagett (1966) shared the same opinion. Thoracotomy was indicated in most cases when the diagnosis was uncertain.

Bentley and Lister (1965) presented a case that required reoperation because of intestinal obstruction after a thoracic procedure. At the time of the second operation they found the hernial sac bilateral and gut strangulated on the other side from the thoracotomy. They advocated the abdominal approach as the one by which the surgeon can confirm that the lesion is bilateral.

We propose a preperitoneal subxiphoid approach (Fig. 5) because it allows freeing of the pleural adhesions to the sac by an extrapleuroperitoneal route. It avoids the wide incision of a laparotomy. A small incision in the neck of the peritoneal sac should be sufficient to return the herniated viscera into the peritoneal cavity. The skin incision is small and can be converted if required to an anterior thoracotomy incision. We used this approach in our last patient.

We are indebted to Drs. L. Garcia-Marrero and J. Esteban of the Department of Radiology, Centro Hospitalario 'La Fé' for their help.

\section{REFERENCES}

Bentley, G., and Lister, J. (1965). Retrosternal hernia. Surgery, 57, 567.

Boyd, D. P. (1961). Diaphragmatic hernia through the foramen of Morgagni. Surgical Clinics of North America, 41, 839.

Chin, E. F., and Duchesne, E. R. (1955). Parasternal defect. Thorax, 10, 214.

Comer, T. P., and Clagett, O. T. (1966). Surgical treatment of hernia of the foramen of Morgagni. Journal of Thoracic and Cardiovascular Surgery, 52, 461.

Denisart, P. (1951). De la variété rétro-costo-xiphoidienne des hernies diaphragmatiques. Journal de Chirurgie, 67, 407.

Dor, J., and Eymery (1956). Hernia diaphragmatique anteriéure du côté gauche. Poumon et Coeur, 12, 375.

Harrington, S. W. (1951). Clinical manifestations and surgical treatment of congenital types of diaphragmatic hernia. Review of Gastroenterology, 18, 243.

Kelly, K. A., and Bassett, D. L. (1964). An anatomic reappraisal of the hernia of Morgagni. Surgery, 55, 495.

Larrey, D. J. (1829). Nouveau procédé opératoire pour ouvrir le péricarde dans le cas d'un épanchement de fluide dans sa cavité. Clinique Chirurgicale, vol. 2, p. 303. Gabon, Paris.

Martinez, Rodriguez, E. (1973). Hernia diafragmática de ? Morgagni. Revista Española de las Enfermedades del O Aparato Digestivo, 39, 77.

Morgagni, J. B. (1761). De sedibus, et causis morborum, vol. 2, p. 322. Venice, Typographia Remondiniana.

Thomas, T. V. (1972). Subcostosternal diaphragmatic 0 hernia. Journal of Thoracic and Cardiovascular Surgery, 63, 279.

Warwick-Brown, R. (1953). Case of retrosternal diaphrag- 을. matic hernia. Thorax, 8, 162.

\section{ADDENDUM}

A further two patients were operated upon, making a total of 11 reported cases in our series. The first $\sigma$ was a 67-year-old woman with mild gastrointestinal and respiratory symptoms, the second a 15-year-old boy with symptoms of upper intestinal obstruction. $\&$ Both were operated on through a preperitoneal subxiphoid approach and the hernia was right-sided. The sac contained the colon accompanied by the omentum in the first case and by the gastroduodenal junction in the second, which explained the continuous vomiting. Both patients made an uneventful recovery $\sigma$ and were discharged on the seventh postoperative day. 